\title{
GROUPS WHICH ADMIT AUTOMORPHISMS IN WHICH EXACTLY THREE-FOURTHS OF THE OPERATORS CORRESPOND TO THEIR INVERSES
}

\author{
BY G. A. MILLER
}

It is well known that a necessary and sufficient condition that a given group $G$ be abelian is that each of the operators of $G$ correspond to its inverse in one of the possible automorphisms of $G$, and that in an automorphism of an abelian group all the operators must correspond to their inverses if more than half of them satisfy this condition. Moreover, it is known that in a non-abelian group at most three-fourths of the operators correspond to their inverses in a possible automorphism. The object of the present article is to determine some fundamental properties of the category of groups which separately admit an automorphism in which exactly three-fourths of the operators correspond to their inverses. Such an automorphism will be called a threefourths automorphism.

Suppose that $G$ is a group for which a three-fourths automorphism has been established, and that $s_{1}$ and $s_{2}$ are any two of its operators which are both non-commutative and correspond to their inverses in this automorphism, while $s_{\alpha}$ is any operator of $G$ which corresponds to its inverse in the same automorphism. A necessary and sufficient condition that $s_{1} s_{\alpha}$ correspond to its inverse in this automorphism is that $s_{1}$ and $s_{\alpha}$ be commutative. Since $s_{1} s_{\alpha}$ must correspond to its inverse for at least two-thirds of the possible values of $s_{\alpha}$ it results that $s_{1}$ is commutative with exactly one-half of the operators of $G$, and that these operators constitute an abelian subgroup of $G$ since all of its operators correspond to their inverses in an automorphism. Since an abelian subgroup of index 2 must also correspond 
to $s_{2}$, and these two subgroups are distinct, it results that $G$ is a non-abelian group which has more than one abelian subgroup of index 2. It therefore contains exactly three such subgroups and has a commutator subgroup of order 2 .

In the given three-fourths automorphism of $G$ all of the operators of two of its abelian subgroups of index 2 correspond to their inverses while only half of those of the third such subgroup satisfy the same condition. The remaining operators of this third abelian subgroup obviously correspond in this automorphism to their inverses multiplied by the commutator of order 2. Moreover, the order of $G$ is divisible by 8 , and $G$ is the direct product of an abelian group of odd order and a group of order $2^{m}$ which admits a three-fourths automorphism. It is obvious that every such direct product admits exactly three three-fourths automorphisms, one for each of its pairs of abelian subgroups of index 2 . Hence the following theorem has been established.

THEOREM 1. If a group admits one three-fourths automorphism it admits exactly three such automorphisms, and it is the direct product of a group of order $2^{m}$ which has this property and an abelian group of odd order. Moreover, every such direct product admits a three-fourths automorphism.

Each of the three three-fourths automorphisms of $G$ is of order 1 or 2 , and the three products of these automorphisms in pairs are the three non-identity inner automorphisms of $G$. As these products are of order 2 the three three-fourths automorphisms of $G$ are commutative and generate a group which is either of order 4 or of order 8 . In the former case this group is the group of inner automorphisms of $G$, and each of the three three-fourths automorphisms of $G$ is an inner automorphism of $G$, which may be the identity automorphism for one of them, while in the latter case none of the threefourths automorphisms is an inner automorphism and their continued product is the characteristic outer automorphism of $G$ in which each of the operators of the central of $G$ corresponds to its inverse while each of the remaining operators of $G$ corresponds to its inverse multiplied by the com- 
mutator of order 2 contained in $G$. This continued product is obviously also a characteristic automorphism when the three-fourths automorphisms are inner automorphisms of $G$.

A necessary and sufficient condition that one of the three three-fourths automorphisms of $G$ be the identity automorphism is that exactly one-fourth of the operators of $G$ have orders which exceed 2 . Hence this condition characterizes the groups which are direct products of the octic group and an abelian group of order $2^{m}$ and of type $(1,1,1$, ...).* The continued product of the three three-fourths automorphisms in this case is the characteristic inner automorphism of order 2 in which all the operators of the subgroup of index 2 generated by the operators of order 4 contained in $G$ correspond to themselves. The other two threefourths automorphisms in this case are the two non-characteristic inner automorphisms of $G$. In the other case where the three three-fourths automorphisms of $G$ generate a group of order 4 , they must therefore all be inner automorphisms of order 2 and hence $G$ must be the Hamiltonian group of order $2^{m}$. That is, $G$ must be the direct product of the quaternion group and the abelian group of order $2^{m-3}$ and of type $(1,1,1, \cdots)$.

From the preceding paragraph it results that when the three three-fourths automorphisms of $G$ generate a group of order 4 then $G$ is the direct product of an abelian group of order $2^{\alpha}$ and of type $(1,1,1, \cdots)$, and either the octic group or the quaternion group. When $G$ is the direct product of any abelian group which is not of the given type and one of the two non-abelian groups of order 8 its three threefourths automorphisms will generate a group of order 8 and of type $(1,1,1, \cdots)$, and when the three three-fourths automorphisms of $G$ generate such a group this will also be the case of the direct product of $G$ and any abelian group whatever. While every group which admits three-fourths automorphisms is the direct product of its Sylow subgroups,

* G. A. Miller, Annals of Mathematics, vol. 7 (1906), p. 55. 
and only one of these Sylow subgroups is non-abelian, this non-abelian subgroup $H$ of order $2^{m}$ may itself be the direct product of an abelian group and a non-abelian group. This can obviously not be the case when it has only two independent generators. We shall prove that it is always the case when it has more than three such generators and that it may or may not be the case when it has exactly three such generators.

Let $H_{0}$ be the central of $H$ and suppose the operators of $H$ are arranged in co-sets with respect to $H_{0}$. We select $s_{1}, s_{2}$ from two distinct co-sets so that their orders are as low as possible, and when their orders are different we assume that the order of $s_{1}$ is the larger. If $H_{0}$ contains any operator whose order is equal to that of $s_{1}$, we select all the operators whose orders are at least equal to this order in any given reduced set of independent generators of $H_{0}$. The group generated by these operators has only the identity in common with the group generated by $s_{1}$, since $s_{1}$ is an operator of lowest order in the co-set to which it belongs. We therefore adjoin $s_{1}$ to the operators already selected in the given set of independent generators of $H$. These operators when $s_{1}$ is replaced by $s_{1}{ }^{2}$ appear in a reduced set of independent generators of $H_{0}$ if the order of $s_{1}$ exceeds 2 . If the order of $s_{1}$ is 2 the order of $s_{2}$ is also 2 and the non-identity commutator of $H$ is an operator of order 2 in the group generated by the selected set of independent generators of $H_{0}$. If this operator is not a power of one of the operators of this set they may evidently be re-selected in such a way that it is such a power, and $H$ is the direct product of the group generated by $s_{1}, s_{2}$ and this operator, and the group generated by the rest of the operators in the given set of independent generators of $H_{0}$. In what follows we shall therefore assume that the order of $s_{1}$ exceeds 2 and hence $s_{1}^{2}$ is an operator in a reduced set of independent generators of $H_{0}$.

When the order of $s_{2}$ is equal to that of $s_{1}$ we may add also $s_{2}{ }^{2}$ to these generators and augment the set thus obtained by other operators in a reduced set of independent generators of $H_{0}$. The only case when this cannot be done is when $s_{1}{ }^{2}=s_{2}{ }^{2}$ 
and hence the order of $s_{1}$ is 4 , but this order may also be 4 when $s_{1}{ }^{2} \neq s_{2}{ }^{2}$. In the former case $s_{1}, s_{2}$ generated the quaternion group while they generate a group of order 16 or of order 32 in the latter case. When the order of $s_{2}$ is less than the order of $s_{1}$ we adjoin to the operators of the given set of reduced independent generators of $H_{0}$ others, if any exist, whose orders are equal to or greater than that of $s_{2}$. We thus obtain a set of reduced independent generators of $H_{0}$ when the order of $s_{2}$ is 2 . When this order exceeds 2 we add $s_{2}{ }^{2}$ to this set and complete a set of reduced independent generators of $H_{0}$ in the usual way. Hence we may state the following theorem.

THEOREM 2. If $H$ is any non-abelian group of order $2^{m}$ which involves more than one abelian subgroup of index 2 , then it is possible to select all the operators of a set of independent generators of $H$ in such a way that only one pair of them is composed of non-commutative operators and that the commutator of order 2 is a power of one of these independent generators.

From this theorem it follows that when the commutator of order 2 in $H$ is a power of $s_{1}$ or of $s_{2}$, then $H$ is the direct product of the group generated by $s_{1}, s_{2}$, and the group generated by all the remaining operators of the given set of independent generators of $H$. In all other cases we adjoin to $s_{1}, s_{2}$ the independent generators whose power is the commutator of order 2 , and $H$ is then the direct product of the group generated by these three operators and the group generated by the remaining operators in the given set of independent generators of $H$. This proves the following theorem.

THEOREм 3. Whenever $H$ has more than three independent generators it is the direct product of an abelian group and a nonabelian group which has at most three independent generators of $H$ for its independent generators.

When it has three such generators they may be so selected that one of them appears in the central of $H$.

Whenever a non-abelian group $G$ involves three abelian subgroups of index 2 then either all of these subgroups are 
conjugate under the group of isomorphisms of $G$ or two of them are thus conjugate while the third is a characteristic subgroup of $G$. Hence it results that the group of inner isomorphisms of such a group involves at most one characteristic operator of order 2 under its group of isomorphisms. When the three three-fourths automorphisms of $G$ generate a group of order 8 it therefore results that this group may involve either only one or three operators of order 2 which are characteristic under the group of isomorphisms of $G$. If it involves only one such operator its other six operators of order 2 are composed of two sets of three conjugate operators under this group of isomorphisms. In the other possible case the four non-characteristic operators of order 2 appear in two sets of two conjugates under the same group.

It was noted above that when the three three-fourths automorphisms of $G$ generate a group of order 4 , then the central of $G$ can involve only operators of order 2 besides the identity. If these automorphisms generate a group of order 8 and the central of $G$ is generated by operators of order 2 then the three abelian subgroups of index 2 under $G$ are either all of type $(2,1,1, \cdots)$, or two of them are of this type while the third is of type $(1,1,1, \cdots)$. In the latter case an operator $t$ which transforms $G$ according to a three-fourths automorphism and has its square in $G$ gives rise to a group which has an abelian subgroup of index 2 . This is also obviously the case when $t$ transforms $G$ according to an operator in its group of inner isomorphisms but it cannot happen when the central of $G$ involves operators whose orders exceed 2. This gives rise to the following theorem.

THEOREM 4. There is one and only one group $G$ of order $2^{m}$, $m>4$, which satisfies the two conditions that its central involves no operator whose order exceeds 2 and that it appears in a group of order $2^{m+1}$ involving operators which transform exactly three-fourths of the operators of $G$ into their inverses but involving no abelian subgroup of index 2. 
Since the Hamiltonian groups of order $2^{m}$ constitute such a well known and important category of groups it may be of interest to note here that it results from the preceding developments that these groups are characterized by the facts that they admit three-fourths automorphisms and that the continued product of these three automorphisms is the identity. This category is also characterized by the fact that it is composed of the groups whose three three-fourths automorphisms are inner automorphisms of order 2. The entire category of Hamiltonian groups may be similarly characterized by the fact that it is composed of the groups whose centrals involve no operators of order 4 and which admit such three three-fourths automorphisms that in their continued product all the operators of the central correspond to their inverses and at least one operator in each central co-set corresponds to itself.

The University of Illinois

\section{THE ROLE OF DIRECTION IN THE VARIATIONS OF A DEFINITE INTEGRAL IN SPACE}

\section{BY LOUIS BRAND}

1. Introduction. In the space problem of the calculus of variations, whether treated in the parametric form as by Bliss and Mason, $\dagger$ or in terms of angle as by Rider $\ddagger$ or Sakellariou, $\$$ the Euler equations are three in number but connected by a relation. Thus but two of the equations are independent. The object of this paper is to show that two independent Euler equations arise from variations parallel

* Presented to the Society, April 16, 1927.

$\dagger$ Mason, M. and Bliss, G. A., The properties of curves in space which minimize a definite integral, Transactions of this Society, vol. 9 (1908), pp. 440-466.

$\ddagger$ Rider, P. R., The space problem of the calculus of variations in terms of angle, American Journal of Mathematics, vol. 39 (1917), pp. 241-256.

$\S$ Sakellariou, N., The space problem of the calculus of variations, Palermo Rendiconti, vol. 44 (1920), pp. 53-68. 